\title{
Empirical modeling and control for spray drying of orange juice powder
}

\begin{abstract}
Spray drying is a removal of moisture from liquid feed by breaking into droplets in a hot medium to convert into powder form. In order to ensure the product quality is at the desired specification, a good control system and good understanding on the dynamic behavior should be considered. The aims of this study are to develop empirical model of spray drying process and improve the process by implementation of PI controller. A nozzle atomizer spray dryer, Lab-Plant SD 05 Laboratory Scale Spray Dryer was used. The liquid feed was Sunquick Concentrated Orange Juice and DE 10-15 maltodextrin as the drying agent. The effects of inlet air temperature and maltodextrin concentration on final moisture content and outlet air temperature were investigated. From investigation, the effect of inlet air temperature on moisture content and outlet air temperature was greater than maltodextrin concentration. Thus, inlet air temperature was selected as manipulated variable. For modeling, the model obtained can be represented as first order process with time delay (FOPTD). In order to improve the process, the model obtained was used in simulation studies to determine the suitable tuning method by PI controller. The PI controllers were tuned by direct synthesis, min IAE method and Cohen-coon. From the observation, direct synthesis method is the most suitable tuning method for PI controller in spray drying process.
\end{abstract}

Keyword: Empirical modeling; Spray drying; Maltodextrin 